\title{
Parthenolide inhibits osteoclast differentiation and bone resorbing activity by down-regulation of NFATc1 induction and c-Fos stability, during RANKL-mediated osteoclastogenesis
}

\author{
Ju-Young Kim ${ }^{1, \#}$, Yoon-Hee Cheon ${ }^{2,3, \#}$, Kwon-Ha Yoon ${ }^{1,4}$, Myeung Su Lee ${ }^{1,5,6, *}$ E Jaemin Oh Ja,3,5,* $^{1,3}$ \\ ${ }^{1}$ Imaging Science-based Lung and Bone Diseases Research Center, ${ }^{2}$ Department of Anatomy, ${ }^{3}$ BK21plus Program and Department of Smart \\ Life-care Convergence Graduate School, ${ }^{4}$ Department of Radiology, ${ }^{5}$ Institute for Skeletal Disease, ${ }^{6}$ Department of Rheumatology, Wonkwang \\ University School of Medicine, Iksan 570-749, Korea
}

\begin{abstract}
Parthenolide, a natural product derived from Feverfew, prevents septic shock and inflammation. We aimed to identify the effects of parthenolide on the RANKL (receptor activator of NF- $\mathrm{KB}$ ligand)-induced differentiation and bone resorbing activity of osteoclasts. In this study, parthenolide dose-dependently inhibited RANKL-mediated osteoclast differentiation in BMMs, without any evidence of cytotoxicity and the phosphorylation of p38, ERK, and IKB, as well as IKB degradation by RANKL treatment. Parthenolide suppressed the expression of NFATc1, OSCAR, TRAP, DC-STAMP, and cathepsin K in RANKL-treated BMMs. Furthermore, parthenolide down-regulated the stability of c-Fos protein, but could not suppress the expression of c-Fos. Overexpression of NFATc1 and c-Fos in BMMs reversed the inhibitory effect of parthenolide on RANKL-mediated osteoclast differentiation. Parthenolide also inhibited the bone resorbing activity of mature osteoclasts. Parthenolide inhibits the differentiation and bone-resolving activity of osteoclast by RANKL, suggesting its potential therapeutic value for bone destructive disorders associated with osteoclast-mediated bone resorption. [BMB Reports 2014; 47(8): 451-456]
\end{abstract}

\section{INTRODUCTION}

Osteoporosis is a fatal problem that is characterized by fragile bone and impaired bone quality. Bone fracture results in in-

*Corresponding authors. Myeung Su Lee, Tel: +82-63-859-2661; Fax: +82-63-852-2661; E-mail: ckhlms@wku.ac.kr, Jaemin Oh, Tel: +82-63-850-6757; Fax: +82-63-852-9115; E-mail: jmoh@wku. ac.kr, ckhlms@wonkwang.ac.kr

${ }^{\#}$ The first two authors contributed equally to this work.

http://dx.doi.org/10.5483/BMBRep.2014.47.8.206

Received 12 September 2013, Revised 17 October 2013, Accepted 28 November 2013

Keywords: Bone disorders, c-Fos, NFATc1, Osteoclase differentiation, Parthenolide creased mortality, and poor quality of life in elderly people (1, 2 ). As the population of elderly people increases, the incidence of osteoporosis, is expected to rise, leading to increased risk of fracture. Therefore, in patients with diseases related to excessive bone loss, the key for sustaining the quality life is the regulation of bone resorption.

Osteoclasts are bone resorbing multinucleated giant cells that are differentiated from hematopoietic stem cells, upon stimulation by two essential cytokines, receptor activator of NF- $\mathrm{KB}$ ligand (RANKL), and macrophage-colony stimulating factor (M-CSF) (3). They play a central role in bone destructive disorders: RANKL promotes osteoclast formation from osteoclast precursors, while M-CSF supports the proliferation and survival of precursor cells during osteoclast differentiation (4). For successful osteoclast differentiation, gene expression controlled by multiple signaling pathways is required, including NF- $\kappa \mathrm{B}, \mathrm{MAP}$ kinases, Akt, and two major transcription factors, c-Fos and NFATc1, are required (5-9).

Numerous natural products have been widely used as traditional medicine, and as therapeutic agents. Since they have fewer side effects, and are more suitable for long-term use than synthetic drugs, several natural products have long been used to prevent and treat osteoporosis. These natural products contain numerous chemical compounds that have been reported to suppress osteoclast differentiation and activity, leading to the prevention of in vivo bone loss $(10,11)$.

Sesquiterpene lactones, found in Asteraceae and Compositae species, have been used as folk remedies for inflammation, arthritis, and tumors. Parthenolide, one of the major sesquiterpene lactones found in the medicinal plant Feverfew, is an active compound with anti-inflammatory properties. Parthenolide has various effects, such as protection from septic shock, proteinuria, and inflammatory renal diseases. In particular, parthenolide blocks LPS-mediated osteolysis, through suppression of NF-KB activation, suggesting that it may be useful for the treatment of infection-mediated bone loss disorders (12). However, the effects of parthenolide on the mechanism of RANKL-induced osteoclast differentiation and activity have not been thoroughly studied. In the present study, we investigated 
the effects of parthenolide on the signaling pathways that are involved in osteoclast differentiation and activation.

\section{RESULTS}

\section{Parthenolide suppresses RANKL-mediated osteoclast differentiation in BMMs}

Fig. 1A shows the structure of parthenolilde used in this study. Primary bone marrow macrophages (BMMs) in the presence of RANKL and M-CSF were treated with, or without, various concentrations of parthenolide, to identify the efficacy of parthenolide on RANKL-mediated osteoclastogenesis. While RANKL differentiated the BMMs of the control group into TRAP-positive immature mono-nucleated and mature multi-nucleated osteoclasts, parthenolide dose-dependently decreased the formation of both TRAP-positive mono-nucleated (early stage of osteoclastogenesis) and multi-nucleated (late stage of osteoclastogenesis) osteoclasts (Figs. 1B-D). Next, we conducted the XTT assay, to exclude the possibility that the inhibitory effect of parthenolide on osteoclastogenesis was due to reduced viability and/or proliferation of the osteoclast precursor cells. Parthenolide had no cytotoxic effects, and did not affect cell proliferation, at doses that effectively inhibited osteoclast differentiation (Fig. 1E).

Parthenolide inhibits RANKL-mediated phosphorylation of p38, ERK, and IKB, as well as degradation of IKB in BMMs RANKL activates several signal transducers involved in osteo-<smiles>C=C1C(=O)O[C@H]2C1CC/C(C)=C/CCC2(C)O</smiles>

C
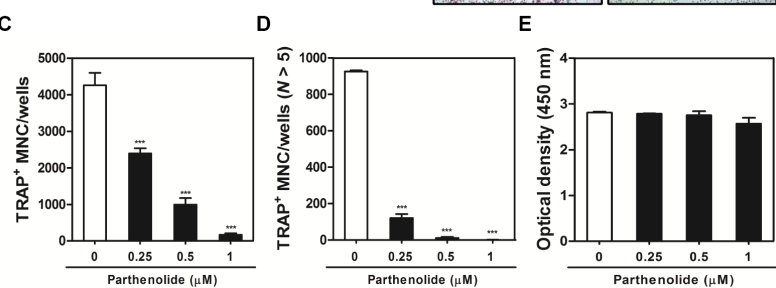

Fig. 1. Effects of parthenolide on RANKL-induced osteoclast differentiation in BMMs. (A) Structure of parthenolide. (B) BMMs were cultured with M-CSF $(30 \mathrm{ng} / \mathrm{ml})$ and RANKL $(100 \mathrm{ng} / \mathrm{ml})$, in the presence, or absence, of parthenolide, at the indicated concentrations. After 4 days, the cells were fixed and stained for TRAP. (C) TRAP-positive mono-nucleated cells $\left(\mathrm{TRAP}^{+} \mathrm{MNCs}\right.$ ) and (D) TRAPpositive multinucleated osteoclasts $\left(\mathrm{TRAP}^{+} \mathrm{MNCS}(\mathrm{N}>5)\right.$ ) with more than 5 nuclei were counted. ${ }^{* * * P}<0.001$ versus control. (E) BMMs were cultured with parthenolide for 3 days at the indicated concentrations, in the presence of M-CSF $(30 \mathrm{ng} / \mathrm{ml})$. Cell viability was determined by XTT assay. clastogenesis, including p38, JNK, ERK and Akt, as well as $\mathrm{NF}-\kappa \mathrm{B}$, which is recognized as a key transcriptional factor for osteoclast differentiation. The effects of parthenolide on RANKL-induced signaling pathways were investigated, to understand the inhibitory mechanism of parthenolide, during RANKL-induced osteoclast differentiation. After the osteoclast precursors were pretreated with parthenolide for $1 \mathrm{~h}$, and stimulated with RANKL at the indicated time, we examined several signaling pathways, and found that phosphorylation of p38, ERK, and IKB were significantly inhibited by parthenolide, as well as degradation of IKB by RANKL (Fig. 2).

Parthenolide inhibits osteoclast differentiation through downregulation of RANKL-induced NFATc1 induction and c-Fos stability, and ectopic overexpression of c-Fos or NFATc1 reverses the parthenolide-mediated inhibition of osteoclast differentiation

The c-Fos and NFATc1 play an essential role in osteoclast differentiation, and regulate the expression of osteoclast-related genes. To assess whether parthenolide has any effects on the induction of c-Fos and NFATc1, the expression of c-Fos and NFATc1 by RANKL was examined. Osteoclast precursors were treated with parthenolide, and further stimulated with RANKL at the indicated time points. We found that in response to RANKL, mRNA and protein levels of c-Fos and NFATc1 increased. Increased protein and mRNA expression of NFATc1 were significantly inhibited by parthenolide (Figs. 3A, B). Parthenolide decreased the increased amount of c-Fos protein by RANKL, without any marked changes in c-Fos mRNA expression (Figs. 3A, B). Therefore, we evaluated whether parthenolide enhances the degradation of c-Fos protein. After $48 \mathrm{~h}$ post-transfection with c-Fos, treatment with $\mathrm{CHX}$, an inhibitor of protein synthesis, decreased the amount of c-Fos protein, compared to control (Fig. 3C); and additional parthenolide

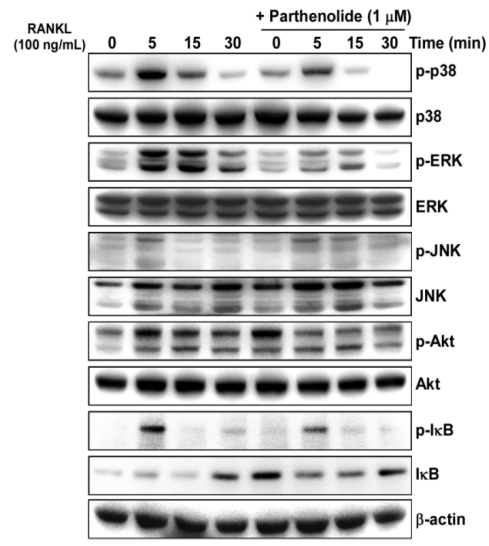

Fig. 2. Parthenolide inhibits RANKL-mediated early signaling. BMMs were pretreated with or without parthenolide $(1 \mu \mathrm{M})$ for $1 \mathrm{~h}$, prior to RANKL stimulation at the indicated time. Lysates were analyzed by western blot analysis with the indicated antibodies. 
treatment accelerated the decrease of c-Fos protein levels. Furthermore, the effect of parthenolide was reversed by co-treatment with selective proteasome inhibitors, namely, MG132 or ALLN. These results suggest that proteasome-mediated degradation is related to the parthenolide-induced reduction in C-Fos protein (Fig. 3C). To examine whether ectopic c-Fos or NFATc1 expression can reverse the effect of parthenolide on osteoclast differentiation, we used a retroviral system to overexpress c-Fos and NFATc1 in BMMs. Overexpression of c-Fos or NFATc1 significantly reversed the parthenolide-mediated inhibition of osteoclast differentiation (Figs. 3D, E). These results indicate that down-regulation of protein levels of c-Fos and NFATC1 is responsible for the inhibitory effect of parthenolide, during RANKL-mediated osteoclastogenesis in BMMs.
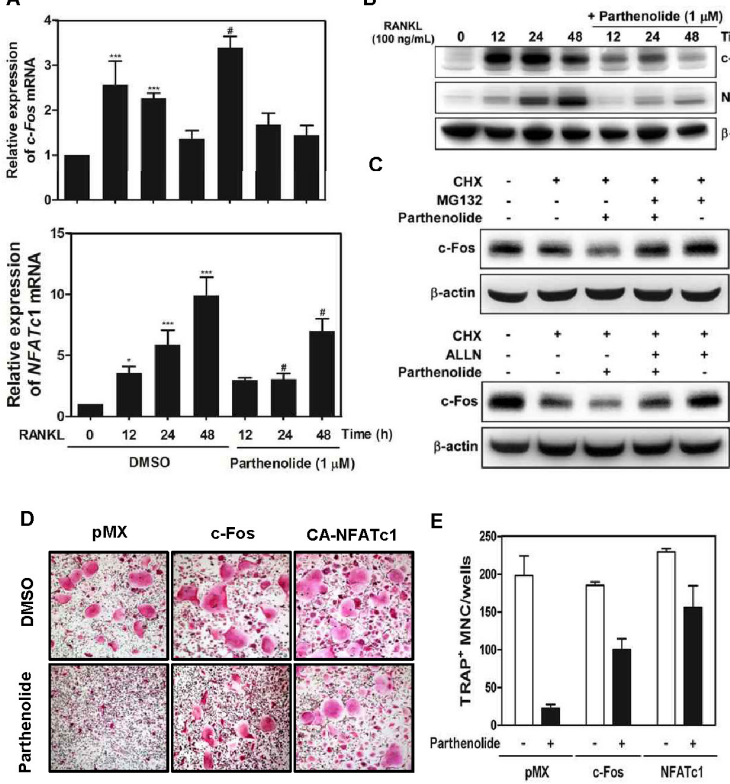

Fig. 3. Parthenolide inhibits osteoclast differentiation through suppression of RANKL-induced NFATC1 induction and c-Fos stability, and overexpression of c-Fos or NFATc1 rescues osteoclast differentiation, despite the presence of parthenolide. (A) BMMs were stimulated with RANKL and M-CSF in the presence, or absence, of parthenolide $(1 \mu \mathrm{M})$, for the indicated times. The mRNA expression levels were evaluated by RT-PCR. (B) Effects of parthenolide on protein expression levels of c-Fos and NFATc1 were evaluated by western blot analysis. (C) Plat E cells were transfected with c-Fos $(2 \mu \mathrm{g})$, and then treated with DMSO or parthenolide $(1 \mu \mathrm{M})$. After $48 \mathrm{~h}$ of transfection, $5 \mu \mathrm{M}$ MG132 or $20 \mu \mathrm{M}$ ALLN, and $2 \mu \mathrm{g} / \mathrm{ml} \mathrm{CHX}$ were added to the cultures for $4 \mathrm{~h}$, before harvest. c-Fos protein levels were detected by western blot analysis. (D, E) BMMs were infected with retroviruses expressing pMX-IRES-EGFP, pMX-c-Fos-EGFP, and pMX-CA-NFATc1-EGFP. (D) Infected BMMs were cultured for $12 \mathrm{~h}$ or $48 \mathrm{~h}$, and western blot analysis was performed for c-Fos or NFATc1 expression levels, respectively. (E) Infected cells were cultured with or without parthenolide in the presence of M-CSF and RANKL, for 4 days. After culturing, the cells were stained for TRAP. (F) TRAP-positive multinucleated cells (TRAP ${ }^{+}$MNCs) were counted.
Parthenolide inhibits mRNA expression of OSCAR, TRAP, DC-STAMP, and cathepsin K by RANKL, and inhibits the bone resorbing activity of mature osteoclasts

During osteoclastogenesis, the expression of OSCAR, TRAP, DC-STAMP, and cathepsin $\mathrm{K}$ is regulated by NFATC1. We examined whether parthenolide regulates the expression of OSCAR, TRAP, DC-STAMP, and cathepsin K, which play essential roles during RANKL-mediated osteoclast differentiation. Parthenolide suppressed mRNA expression of OSCAR, TRAP, DC-STAMP, and cathepsin K by RANKL (Fig. 4A). These data suggest that, through suppression of NFATc1, parthenolide may inhibit RANKL-induced mRNA expression of OSCAR, TRAP, DC-STAMP, and cathepsin K. Next, we examined whether parthenolide has the potential to inhibit the bone resorbing activity of mature osteoclasts. After mature osteoclasts were cultured on hydroxyapatite-coated plates for $24 \mathrm{~h}$, we observed the resolved area of the plate under a microscope.
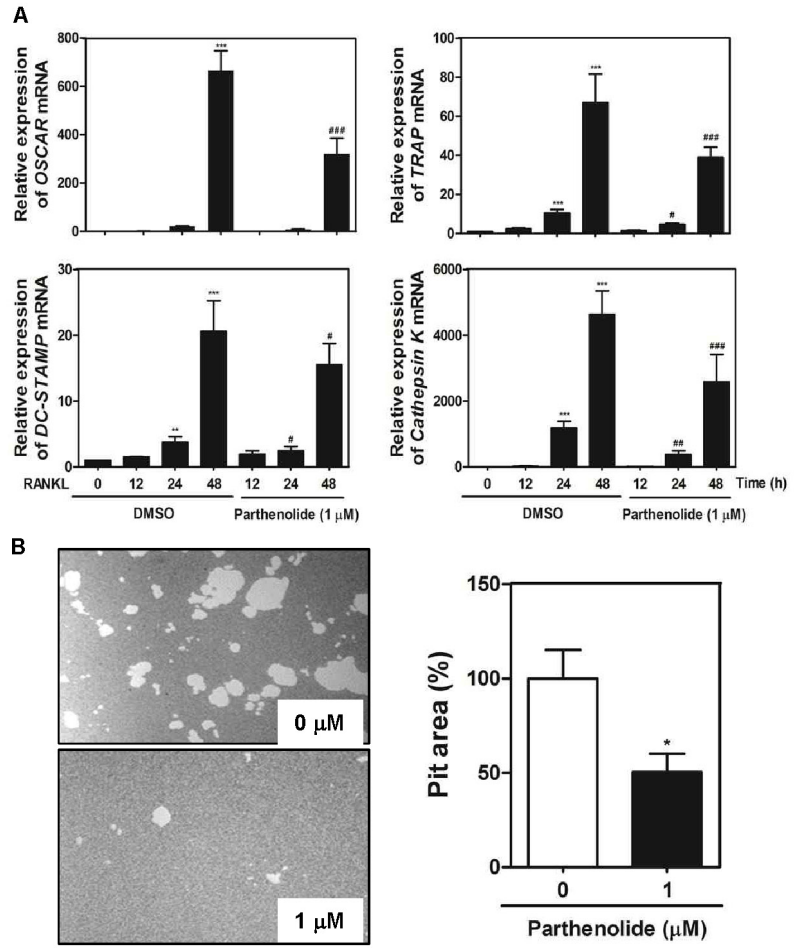

Fig. 4. Parthenolide inhibits OSCAR, TRAP, DC-STAMP, and cathepsin $\mathrm{K}$ expression and bone resorption. After pretreatment with the DMSO or parthenolide $(1 \mu \mathrm{M})$ for $1 \mathrm{~h}, \mathrm{BMMs}$ were treated with RANKL for the indicated time. (A) Then, mRNA expression levels were analyzed by real time RT-PCR. Parthenolide inhibits osteoclastic bone resorption. Mature osteoclasts were seeded on hydroxyapatite-coated plates and treated for $24 \mathrm{~h}$ with parthenolide $(1 \mu \mathrm{M})$. (B) Attached cells on the plates were removed, and photographed under a light microscope (left). Pit areas were quantified using Image J (right). ${ }^{* * P}<0.01, * * * \mathrm{P}<0.001$ versus control at $0 \mathrm{~h} ;{ }^{\#} \mathrm{P}<0.05,{ }^{\# \#} \mathrm{P}<0.01$, \#\# $\mathrm{P}<0.001$ versus control at each time. ${ }^{*} \mathrm{P}<0.05$ versus control. 
Parthenolide decreased the area of resolved pits by mature osteoclasts, while the vehicle could not suppress the increase in the area and number of resolved pits by mature osteoclasts (Fig. 4B), suggesting that parthenolide inhibits the bone resorbing activity of mature osteoclasts.

\section{DISCUSSION}

Bone fractures by osteoporosis can result in significant problems, including high mortality, and economic burden in the elderly population. To improve the quality of life in patients with excessive bone-resolving disorders, prevention and treatment of bone loss and fracture are critically important. In recent years, the major remedies used for osteoporosis have included estrogen replacement therapy, along with bisphosphonates, selective estrogen receptor modulators (SERM), and calcitonin. However, such therapies are related to severe side effects, including hyperlipidemia, hypertension, endometrial cancer, and gastrointestinal problems $(13,14)$. Thus, efforts have been made to find candidates with fewer side effects, and excellent efficacies for the prevention and/or treatment of bone resorbing disorders. Natural products have received recent attention as potential therapeutic and preventative drugs for human diseases, because they have historically yielded a variety of therapeutic agents. In the present study, we have shown that parthenolides derived from Feverfew effectively inhibited osteoclast differentiation, without any cytotoxicity, at both early and late stages of RANKL-mediated osteoclastogenesis (Fig. 1).

Excessive enhanced RANKL signaling causes bones to turn very fragile. This condition is related to several pathologic disorders, including osteoporosis, periodontitis, and rheumatoid arthritis; thus, down-regulation of RANKL signaling may be a key factor, in the treatment of excessive bone resorbing diseases. Binding of RANKL to its receptor RANK activates various downstream signals, including NF-KB, p38, ERK, JNK, and Akt, to induce the expression of critical genes for osteoclast differentiation. NF- $\mathrm{KB}$ p $50^{--}$and $\mathrm{p} 52^{--}$double knockout mice display defects of osteoclastogenesis and severe osteopetrosis, indicating that NF-KB is an essential factor in osteoclast differentiation $(15,16)$. We found that by suppressing RANKL-mediated IKB $\alpha$ phosphorylation and degradation, parthenolide inhibited activation of NF-KB. Our data is consistent with the previous report that, through down-regulation of NF-KB signaling, parthenolide inhibits LPS-mediated infectious osteolysis. In addition, parthenolide inhibited the RANKL-induced phosphorylation of p38 and ERK (Fig. 2). Since MAP kinases consisting of p38, ERK, and JNK translocate key transcription factors into the nucleus, activation of MAP kinases is essential for osteoclast differentiation. In particular, the importance of p38 signaling in RANKL-induced osteoclast differentiation has been suggested in several reports, and it is considered to be a potential therapeutic agent for bone resorption disorders $(6,17)$. Therefore, our data suggest that through suppression of NF-kB, p38, and ERK, parthenolide inhibits RANKL-mediated osteoclastogenesis.
NFATc1 acts as an essential modulator of RANKL-mediated osteoclast differentiation. Ectopic expression of NFATc1 eliminates the requirement of RANKL for mature osteoclast differentiation, even in the absence of RANKL. Furthermore, NFATc $1^{-1-}$ embryonic stem cells do not successfully differentiate into osteoclasts in response to RANKL (9), suggesting that NFATc1 plays an essential role in RANKL-induced osteoclastogenesis. In addition, c-Fos is a critical element for the induction of NFATc1 by RANKL (8). Therefore, we determined the effects of parthenolide on RANKLinduced mRNA expression and protein levels of c-Fos and NFATc1. In our study, parthenolide down-regulated RANKL-induced protein level and mRNA induction of NFATc1 in a time-dependent manner, while in c-Fos mRNA induction, only the protein level of c-Fos was decreased, without any marked changes. In addition, our protein stability assay showed that down-regulation of c-Fos stability by parthenolide was reversed by treatment with MG132 or ALLN proteasome inhibitors (Figs. 3A-C). These data raise the possibility that parthenolide has dual roles: suppression of NFATc1 induction, and down-regulation of c-Fos stability during RANKL-mediated osteoclast differentiation. Furthermore, ectopic expression of c-Fos or CA-NFATc1 reversed the parthenolide-induced inhibition of osteoclast differentiation, suggesting that down-regulation of c-Fos and NFATc1 is responsible for the inhibitory effects of parthenolide (Figs. 3D, E).

NFATc1 plays an essential role in the regulation of genes in the middle or late stages of osteoclast differentiation mediated by RANKL, and it subsequently induces the expression of osteoclast-specific genes, including OSCAR, TRAP, cathepsin $\mathrm{K}$, and DC-STAMP (18-20). In this study, parthenolide suppressed the induction of OSCAR, TRAP, cathepsin K, and DC-STAMP (Fig. $4 \mathrm{~A})$. Bone resorption is a critical event, which leads to bone destruction and fragility. Osteoclasts perform bone resorption, by resorbing components of the bone matrix. Cathepsin $\mathrm{K}$ is highly expressed in osteoclasts, and is a well- known proteolytic enzyme that degrades the bone matrix (21). Induction of cathepsin $\mathrm{K}$ by NFATC1 is responsible for the degradation of collagen matrix by osteoclasts. In this study, parthenolide inhibited the bone-resorption activity of mature osteoclasts (Fig. 4B); thus, we suggest that parthenolide inhibits the bone resorbing activity of mature osteoclasts, through down- regulation of NFATc1.

In conclusion, parthenolide prevents differentiation and bone-resolving activity of osteoclasts, via down-regulation of p38, ERK, and NF-KB, as well as NFATc1 signaling molecules, and suppression of c-Fos stability. These results suggest that parthenolide has potential therapeutic value for bone destructive disorders that are associated with increased bone resorption.

\section{MATERIALS AND METHODS}

\section{Mice and reagents}

Male, 5-week-old ICR mice were purchased from Damul Science (Daejeon, Korea). All experiments in this study were performed in accordance with the animal experiment guidelines of the Institute Committee of Wonkwang University. 
Recombinant soluble human M-CSF and human RANKL were obtained from PeproTech EC Ltd. (London, UK). Parthenolide, $\mathrm{N}$-acetyl-leu-leu-norleucinal (ALLN), and monoclonal $\beta$-actin antibody were obtained from Sigma (St. Louis, MO, USA). Anti-JNK, anti-phospho-JNK, anti-ERK 1/2, anti-phospho-ERK 1/2, anti-p38, and anti-phospho-p38 antibodies were purchased from Cell Signaling Technology Inc. (Beverly, MA, USA). Anti-c-Fos and anti-NFATc1 antibodies were purchased from Santa Cruz Biotechnology (Santa Cruz, CA, USA). Fetal bovine serum (FBS), $\alpha$-minimum essential medium ( $\alpha$-MEM), and penicillin/streptomycin were purchased from Gibco BRL (Grand Island, NY, USA). Cyclohexamide (CHX) and MG132 were obtained from Calbiochem (San Diego, CA, USA).

\section{Mouse bone marrow macrophage preparation and osteoclast differentiation}

BMCs were obtained from 5-week-old male ICR mice, by flushing the femurs and tibias with $\alpha$-MEM supplemented with $10 \%$ FBS and $1 \%$ antibiotics. To obtain BMMs, the BMCs were cultured in $\alpha$-MEM supplemented with $10 \%$ FBS and M-CSF $(10 \mathrm{ng} / \mathrm{ml})$ for 1 day. Non-adherent cells were transferred to $10 \mathrm{~cm}$ Petri dishes, and further cultured in the presence of M-CSF $(30 \mathrm{ng} / \mathrm{ml})$, for 3 days. After the non-adherent cells were removed, adherent cells were used as BMMs. To generate osteoclasts from the BMMs culture system, BMMs $\left(3.5 \times 10^{4}\right.$ cells/well) were cultured in complete medium containing M-CSF $(30 \mathrm{ng} / \mathrm{ml})$ and RANKL $(100 \mathrm{ng} / \mathrm{ml})$ for 4 days with, or without, parthenolide. The cells were fixed in $3.7 \%$ formalin for 10 min, permeabilized with $0.1 \%$ Triton X-100, and then stained with TRAP (Sigma).

\section{Cell viability assay}

BMMs were seeded in 96-well plates $\left(1 \times 10^{4}\right.$ cells/well), and cultured overnight, then treated with M-CSF $(30 \mathrm{ng} / \mathrm{ml})$, and various concentrations of parthenolide. After 3 days, $50 \mu \mathrm{l}$ XTT reagent were added to each well, and then incubated for $4 \mathrm{~h}$. The optical density of each well was then measured at 450 $n m$, using an ELISA reader.

\section{Real-time RT-PCR}

Total RNA was isolated with QIAzol reagent (Qiagen, Valencia, CA, USA), according to the manufacturer's instructions. Isolated RNA was reverse-transcribed to cDNA, using SuperScript II Reverse Transcriptase (Invitrogen, San Diego, CA, USA). Real-time RT-PCR was conducted in a $20 \mu$ reaction mixture, containing 10 $\mu$ I SYBR Green Premix (Bioneer Co., Daejeon, Korea), 10 pmol forward primer, $10 \mathrm{pmol}$ reverse primer, and $1 \mu \mathrm{g}$ cDNA. The mouse GAPDH gene was used as the internal control. The amplification parameters consisted of initial denaturation at $95^{\circ} \mathrm{C}$ for 5 min, and 40 cycles of 3-step PCR (denaturation at $95^{\circ} \mathrm{C}$ for $1 \mathrm{~min}$, annealing at $60^{\circ} \mathrm{C}$ for $30 \mathrm{sec}$, and extension at $72^{\circ} \mathrm{C}$ for $1 \mathrm{~min}$ ). The data was normalized to $\mathrm{GAPDH}$, and presented as the mean fold change, as compared to controls.

\section{Western blot analysis}

Cell lysates were prepared, using lysis buffer containing $50 \mathrm{mM}$ Tris- $\mathrm{HCl}, 150 \mathrm{mM} \mathrm{NaCl}, 5 \mathrm{mM}$ EDTA, 1\% Triton X-100, 1 mM sodium fluoride, $1 \mathrm{mM}$ sodium vanadate, $1 \%$ deoxycholate, and protease inhibitors. The protein content was measured using a Bio-Rad colorimetric protein assay kit (Bio-Rad Laboratories Inc., Hercules, CA, USA). Equal amounts of proteins $(30 \mu \mathrm{g})$ were run on $8-10 \%$ SDS-PAGE gels, and transferred by electroblotting onto polyvinylidene difluoride membranes (Millipore, Bedford, MA, USA). The membranes were blocked with $5 \%$ nonfat dry milk, and probed for $2 \mathrm{~h}$ with the primary antibodies. After washing with Tris-buffered saline (TBS) containing $0.1 \%$ Tween-20 (TBST), the membranes were incubated for $1 \mathrm{~h}$ with horseradish peroxidase-conjugated sheep anti-mouse or donkey anti-rabbit immunoglobulin antibodies. After washing with TBST, the specific signals were detected, using an enhanced chemiluminescence detection system (Millipore).

\section{Retrovirus preparation and infection}

The retroviral vectors pMX-IRES-EGFP, pMX-cFos-IRES-EGFP, and pMX-constitutively active (CA)-NFATc1-IRES-EGFP packaging were performed by transient transfection of these $\mathrm{pMX}$ vectors into Plat-E retroviral packaging cells, using X-tremeGENE 9 (Roche, Nutley, NJ, USA), according to the manufacturer's protocol. After incubation in fresh medium for 2 days, the culture supernatants of the retrovirus-producing cells were collected. For retroviral infection, nonadherent BMCs were cultured in M-CSF (30 ng/ml) for 2 days. The $B M M s$ were incubated with viral supernatant $\mathrm{pMX}$-IRES-EGFP, pMX-cFos-IRES-EGFP, and pMX-CA-NFATc1-IRES-EGFP virus-producing Plat-E cells, together with polybrene $(10 \mu \mathrm{g} / \mathrm{ml})$ and M-CSF $(30 \mathrm{ng} / \mathrm{ml})$, for $6 \mathrm{~h}$. After infection, the BMMs were induced to differentiate, in the presence of M-CSF $(30 \mathrm{ng} / \mathrm{ml})$ and RANKL $(100 \mathrm{ng} / \mathrm{ml})$, for 4 days.

\section{Bone resorption assay}

Mature osteoclasts were prepared from the BMC and primary osteoblast coculture, as previously described (22). BMC $(1 \times$ $10^{7}$ cells $)$ and primary osteoblasts $\left(1 \times 10^{6}\right.$ cells $)$ were seeded on collagen gel-coated culture dishes, and cultured for 7 days, in the presence of $10^{-8} \mathrm{M} 1,25$-dihydroxyvitamin $D_{3}$ and $10^{-6}$ $\mathrm{M}$ prostaglandin $\mathrm{E}_{2}\left(\mathrm{PGE}_{2}\right)$. The cocultured cells were detached by $0.1 \%$ collagenase treatment at $37^{\circ} \mathrm{C}$ for $10 \mathrm{~min}$, and were then replated on hydroxyapatite-coated plates (Corning, NY, USA). The cells were incubated on the plates with, or without, parthenolide. After $24 \mathrm{~h}$, the cells were removed, and the total resorption pits were photographed and analyzed, using Image-Pro Plus version 4.0 (Media Cybernetics, Silver Spring, MD, USA).

\section{Statistical analyses}

Experiments were conducted separately at least 3 times, and all data are presented as the mean \pm standard deviation (SD). All statistical analyses were performed using SPSS (Korean ver- 
sion 14.0). Student's t-test was used to compare the parameters between 2 groups, while the analysis of variance (ANOVA) test, followed by the Tukey post-hoc test, were used to compare the parameters among the 3 groups. $\mathrm{P}<0.05$ was considered statistically significant.

\section{ACKNOWLEDGEMENTS}

This study was supported by a grant from the Korean Health Technology R\&D Project, Ministry of Health \& Welfare, Republic of Korea (HI12C0110).

\section{REFERENCES}

1. Bible, J. E., Wegner, A., McClure, J. M., Kadakia, R. J., Richards, J. E., Bauer, J. M. and Mir, H. R. (2013) One-year mortality after acetabular fractures in elderly patients presenting to a level-one trauma center. J. Orthop. Trauma [Epub ahead of print].

2. Wang, C. B., Lin, C. F., Liang, W. M., Cheng, C. F., Chang, Y. J., Wu, H. C., Wu, T. N. and Leu, T. H. (2013) Excess mortality after hip fracture among the elderly in Taiwan: A nationwide population-based cohort study. Bone 56, 147-153.

3. Quinn, J. M., Elliott, J., Gillespie, M. T. and Martin, T. J. (1998) A combination of osteoclast differentiation factor and macrophage-colony stimulating factor is sufficient for both human and mouse osteoclast formation in vitro. Endocrinology 139, 4424-4427.

4. Lee, T. H., Fevold, K. L., Muguruma, Y., Lottsfeldt, J. L. and Lee, M. Y. (1994) Relative roles of osteoclast colony-stimulating factor and macrophage colony-stimulating factor in the course of osteoclast development. Exp. Hematol. 22, 66-73.

5. Wong, B. R., Josien, R., Lee, S. Y., Vologodskaia, M., Steinman, R. M. and Choi, Y. (1998) The TRAF family of signal transducers mediates NF-kappaB activation by the TRANCE receptor. J. Biol. Chem. 273, 28355-28359.

6. Matsumoto, M., Sudo, T., Saito, T., Osada, H. and Tsujimoto, M. (2000) Involvement of p38 mitogen-activated protein kinase signaling pathway in osteoclastogenesis mediated by receptor activator of NF-kappa B ligand (RANKL). J. Biol. Chem. 275, 31155-31161.

7. Wong, B. R., Besser, D., Kim, N., Arron, J. R., Vologodskaia, M., Hanafusa, H. and Choi, Y. (1999) TRANCE, a TNF family member, activates Akt/PKB through a signaling complex involving TRAF6 and c-Src. Mol. Cell 4, 1041-1049.

8. Matsuo, K., Galson, D. L., Zhao, C., Peng, L., Laplace, C., Wang, K. Z., Bachler, M. A., Amano, H., Aburatani, H., Ishikawa, $\mathrm{H}$. and Wagner, E. F. (2004) Nuclear factor of activated T-cells (NFAT) rescues osteoclastogenesis in precursors lacking c-Fos. J. Biol. Chem. 279, 26475-26480.

9. Takayanagi, H., Kim, S., Koga, T., Nishina, H., Isshiki, M., Yoshida, H., Saiura, A., Isobe, M., Yokochi, T., Inoue, J., Wagner, E. F., Mak, T. W., Kodama, T. and Taniguchi, T. (2002) Induction and activation of the transcription factor NFATc1 (NFAT2) integrate RANKL signaling in terminal differentiation of osteoclasts. Dev. Cell 3, 889-901.
10. Koyama, T., Nakajima, C., Nishimoto, S., Takami, M., Woo, J. T. and Yazawa, K. (2012) Suppressive effects of the leaf of Terminalia catappa L. on osteoclast differentiation in vitro and bone weight loss in vivo. J. Nutr. Sci. Vitaminol. 58, 129-135.

11. Zhao, Y., Huai, Y., Jin, J., Geng, M. and Li, J. X. (2011) Quinoxaline derivative of oleanolic acid inhibits osteoclastic bone resorption and prevents ovariectomy-induced bone loss. Menopause 18, 690-697.

12. Yip, K. H., Zheng, M. H., Feng, H. T., Steer, J. H., Joyce, D. A. and $\mathrm{Xu}$, J. (2004) Sesquiterpene lactone parthenolide blocks lipopolysaccharide-induced osteolysis through the suppression of NF-kappaB activity. J. Bone Miner. Res. 19, 1905-1916.

13. Knopp-Sihota, J. A., Cummings, G. G., Homik, J. and Voaklander, D. (2013) The association between serious upper gastrointestinal bleeding and incident bisphosphonate use: a population-based nested cohort study. BMC Geriatr. 13, 36.

14. Judd, H. L., Meldrum, D. R., Deftos, L. J. and Henderson, B. E. (1983) Estrogen replacement therapy: indications and complications. Ann. Intern. Med. 98, 195-205.

15. Franzoso, G., Carlson, L., Xing, L., Poljak, L., Shores, E. W., Brown, K. D., Leonardi, A.,, Tran, T., Boyce, B. F. and Siebenlist, U. (1997) Requirement for NF-kappaB in osteoclast and B-cell development. Genes Dev. 11, 3482-3496.

16. Soysa, N. S., Alles, N., Weih, D., Lovas, A., Mian, A. H., Shimokawa, H., Yasuda, H., Weih, F., Jimi, E., Ohya, K. amd Aoki, K. (2010) The pivotal role of the alternative NF-kappaB pathway in maintenance of basal bone homeostasis and osteoclastogenesis. J. Bone Miner. Res. 25, 809-818.

17. Matsumoto, M., Sudo, T., Maruyama, M., Osada, H. and Tsujimoto, M. (2000) Activation of p38 mitogen-activated protein kinase is crucial in osteoclastogenesis induced by tumor necrosis factor. FEBS Lett. 486, 23-28.

18. Kim, J. H., Kim, K., Jin, H. M., Youn, B. U., Song, I., Choi, H. S. and Kim, N. (2008) Upstream stimulatory factors regulate OSCAR gene expression in RANKL-mediated osteoclast differentiation. J. Mol. Biol. 383, 502-511.

19. Lange, A. W. and Yutzey, K. E. (2006) NFATc1 expression in the developing heart valves is responsive to the RANKL pathway and is required for endocardial expression of cathepsin K. Dev. Biol. 292, 407-417.

20. Kim, K., Lee, S. H., Kim, J. H., Choi, Y. and Kim, N. (2008) NFATc1 induces osteoclast fusion via up-regulation of Atp6v0d 2 and the dendritic cell-specific transmembrane protein (DC-STAMP). Mol. Endocrinol. 22, 176-185.

21. Saftig, P., Hunziker, E., Wehmeyer, O., Jones, S., Boyde, A. Rommerskirch, W., Moritz, J. D., Schu, P. and von Figura, K. (1998) Impaired osteoclastic bone resorption leads to osteopetrosis in cathepsin-K-deficient mice. Proc. Natl. Acad. Sci. U. S. A. 95, 13453-13458.

22. Yeon, J. T., Choi, S. W., Park, K. I., Choi, M. K., Kim, J. J., Youn, B. S., Lee, M. S. and Oh, J. (2012) Glutaredoxin2 isoform b (Glrx2b) promotes RANKL-induced osteoclastogenesis through activation of the p38-MAPK signaling pathway. BMB Rep. 45, 171-176. 